\title{
Analysis of a Dipole Antenna Using Maxwell-SCHRÖDINGER Equation
}

\author{
Jinyoung Kim', Jaeyoung Jung', Youngbae Jung ${ }^{2}$ and Changwon Jung ${ }^{1 *}$ \\ ${ }^{1}$ Graduate School of NID Fusion Technology, Seoul National University of Science and Technology \\ ${ }^{2}$ Electronics and Control Engineering, Hanbat National University, Daejeon, Korea \\ 맥스웰-슈뢰딩거 방정식을 이용한 다이폴 안테나 해석 \\ 김진영 ${ }^{1}$, 정재영 ${ }^{1}$, 정영배 ${ }^{2}$, 정창원 ${ }^{*}$ \\ ${ }^{1}$ 서울과학기술대학교 NID융합기술대학원, ${ }^{2}$ 한밭대학교 전자제어공학과
}

\begin{abstract}
We present a quantitative analysis of a dipole antenna and its characteristics from the viewpoint of quantum mechanics. The method makes use of a Maxwell equation used in an existing antenna propagation formula. This includes radiation resistance, input reactance, and antenna efficiency as functions of frequency and antenna length. Particular attention is paid to the Schröodinger equation. We accomplish E-field and H-field analyses of a dipole antenna by combining the Maxwell and Schröodinger wave equations. When comparing the existing Maxwell wave equation with the Schröodinger wave equation, quantum-electric movement is more accurate than using the Maxwell wave equation alone.

요 약 본 논문은 양자역학 관점으로 본 다이폴 안테나의 정량적 분석과 그것의 특성에 관한 논문이다. 분석 방법으로 현존 하는 안테나 전파 방정식에 이용되는 맥스웰 방정식을 사용한다. 이는 안테나의 길이와 주파수에 관한 함수로, 방사저항, 입력 리액턴스, 안테나 효율을 포함한다. 본 논문의 주요 관심사는 슈뢰딩거 방정식이다. 또한 본 논문은 맥스웰과 슈뢰딩거 방정식을 결합하여 다이폴 안테나의 전계와 자계를 해석한다. 현존하는 맥스웰 방정식과 슈뢰딩거 방정식을 비교함으로써, 단일 맥스웰 방정식을 썼을 때 보다 양자-전기 이동의 정확성이 향상됨을 보인다.
\end{abstract}

Key Words : Dipole antenna, Maxwell equation, Schrodinger wave equation, Quantum mechanics

\section{Introduction}

Recently, various studies have conducted numerical analysis and experiments for a more accurate analysis of communication devices at microwave (gigahertz) frequencies. There has been a renewed interest in analyzing antenna propagation characteristics using quantum mechanics in order to take complex antenna environments into account. For instance, unconventional materials with non-linear or homogeneous conductivity and permittivity properties are often combined with novel antennas [1-3]. Also, for an antenna placed in a complex electromagnetic environment, its radiation performance is significantly affected by the surrounding materials or an undesirable radiation source [4,5]. The classical wave equations (e.g., Maxwell equation, acoustic equation) are not sufficient to accurately analyze antenna fields in the

This study was supported by the Research Program funded by the Seoul National University of Science and Technology.

*Corresponding Author : Changwon Jung(Seoul National Univ. of Science and Technology)

Tel: +82-10-9969-4078 email: changwoj@snut.ac.kr

Received April 11, $2014 \quad$ Revised May 7, $2014 \quad$ Accepted May 8, 2014 
aforementioned environments. In order to overcome this limitation, numerical tools based on the method of moment (MoM), finite difference time domain (FDTD), and finite element method (FEM) are frequently used[6-9]. However, there is still demand for closed-form expressions for quick and physically intuitive analysis. For this reason, quantum mechanics has been attempted for antenna analysis [10]. The Schrödinger equation is the key equation in quantum mechanics. It can be effectively relevant to construct a wave function that can satisfactorily describe the probability of finding a freely traveling particle within a given space at a given time. We were introduced by Schrödinger equation for wave equation, unlikely the existing analysis methods.

\section{Dipole antenna analysis by maxwell equation}

\subsection{Electric Field and Magnetic Field Intensity of Dipole Antenna}

Based on the known current distribution, it is straightforward to calculate the radiated electric and magnetic fields. We follow Balanis [11]. For the current distribution of the along the $z^{-}$axis, the electric field in the far field region must be in the $\theta$ direction, and is given by

$$
E_{\theta}=i \eta \frac{k e^{-i k r}}{4 \pi r} \sin \theta\left[\int_{-l / 2}^{+l / 2} I(z) e^{i k z \cos \theta} d z\right]
$$

Here, $\eta$ is the characteristic impedance of free space. For a traditional wire antenna, the current distribution is periodic with a wave vector. Therefore, the distribution of the total field is a product of element factor and space factor. We can express the electric field as

$$
E_{\theta}=i \eta \frac{k e^{-i k r}}{4 \pi r} \sin \theta x\left\{\int_{-/ / 2}^{0} I_{0} \sin \left[k\left(\frac{l}{2}+z\right)\right] e^{i k \cos \theta} d z+\int_{0}^{1 / 2} I_{0} \sin \left[k\left(\frac{l}{2}-z\right)\right] e^{i k \cos \theta} d z\right\}
$$

$$
E_{\theta}=i \eta \frac{I_{0} e^{-j k r}}{2 \pi r}\left[\frac{\cos \left(\frac{k l}{2} \cos \theta\right)-\cos \left(\frac{k l}{2}\right)}{\sin \theta}\right]
$$

The magnetic field component has been obtained in a similar way, as follows.

$$
H_{\phi}=i \frac{I_{0} e^{-j k r}}{2 \pi r}\left[\frac{\cos \left(\frac{k l}{2} \cos \theta\right)-\cos \left(\frac{k l}{2}\right)}{\sin \theta}\right]=\frac{E_{\theta}}{\eta}
$$

2.2 Vector Potential Energy of Dipole Antenna

To find the fields radiated by the current element, it is important to extract vector potential energy [11]. The vector potential $(A)$ is useful in solving for the EM field generated by given harmonic electric current $(J)$. By Maxwell's law, the magnetic flux $(B)$ doesn't always radiate. Therefore, it can be represented as the curl of another vector. (i.e. vector potential $(A)$ )

$$
\nabla \cdot \nabla \times A=0
$$

Where, $A$ is arbitrary vector form. Thus,

$$
B_{a}=\mu H_{a}=\nabla \times A
$$

Therefore, $H_{a}$ can be represented.

$$
H_{a}=\frac{1}{\mu} \nabla \times A
$$

Here, subscript $a$ indicates the field due to the

$A$ potential. Substituting (7) into Maxwell's curl equation

$$
\nabla \times E_{a}=-j \omega \mu H_{a}=-j \omega \nabla \times A
$$

which can be written as

$$
\nabla \times\left[E_{a}+j \omega A\right]=0
$$

It can be represented that

$$
E_{a}+j \omega A=-\nabla \phi_{e}
$$

The scalar function $\phi_{e}$ represents an arbitrary electric scalar potential which is a function of position.

$$
\nabla \times\left(\mu H_{a}\right)=\nabla(\nabla \cdot A)-\nabla^{2} A
$$

Equating Maxwell's equation leads to

After evaluating Equation (2), the result is 


$$
\mu J+j \omega \mu \varepsilon E_{a}=\nabla(\nabla \cdot A)-\nabla^{2} A
$$

Eventually, we obtain wave equation.

$$
\nabla^{2} A+k^{2} A=-\mu J+\nabla(\nabla \cdot A)+\nabla\left(j \omega \mu \varepsilon \phi_{e}\right)
$$

Where, $k^{2}=\omega^{2} \mu \epsilon$.

Following Lorentz equation, we represent magnitude of potential energy.

$$
A=\frac{\mu}{4 \pi} \iiint_{v} J \frac{e^{-j k R}}{R} d v^{\prime}
$$

Therefore, we obtain potential energy of linear dipole antenna.

$$
V(l)=\frac{\mu I_{0}}{4 \pi} \int_{-l / 2}^{+l / 2} \frac{\sin \left[k(l / 2)-\left|z^{\prime}\right|\right]}{r} e^{-i k r} d z^{\prime}
$$

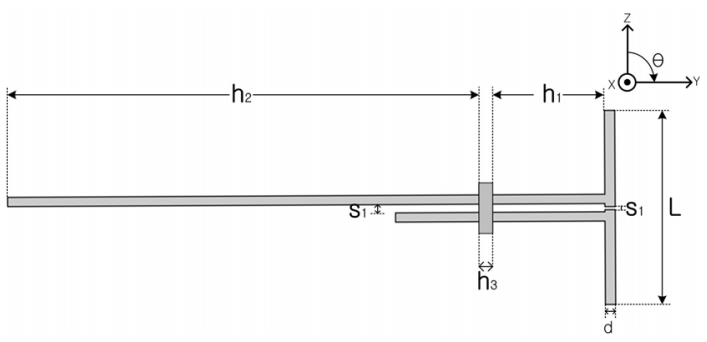

[Fig. 1] Proposed conventional dipole antenna

[Table 1] Dimension of the proposed Dipole Antenna

\begin{tabular}{c|c}
\hline Symbol & Unit(mm) \\
\hline \hline $\mathrm{L}$ & 70 \\
\hline $\mathrm{D}$ & 3.6 \\
\hline $\mathrm{h} 1$ & 40.6 \\
\hline $\mathrm{h} 2$ & 180 \\
\hline $\mathrm{h} 3$ & 4 \\
\hline $\mathrm{s} 1$ & 0.5 \\
\hline $\mathrm{s} 2$ & 3 \\
\hline
\end{tabular}

\section{Introduction of The Schr dinger Equation}

\subsection{The one dimensional time-dependent} Schr dinger equation

The Schrödinger equation is the main equation in the analysis using the quantum mechanical model. The one-dimensional Schrödinger equation is used when the particle interest is confined to one spatial dimension. The particles traveling along the $\mathrm{x}$-axis are assumed. To derive the one-dimensional Schrödinger equation, we start with the total energy equation (i.e., the sum of kinetic and potential energy [12-14].

$$
\frac{p^{2}}{2 m}+U(x)=E_{\text {total }}
$$

Here, $p$ is the momentum of the particle, $m$ is the mass of the particle, $U(x)$ is the potential energy in the combined particle, and $E_{\text {total }}$ is the total energy of the system achieved by combining particles.

The substitution of the dynamical variables with their quantum mechanical operator, which acts on the wave function $\Psi(x, t)$, yields the one-dimensional time-dependent Schrödinger equation:

$$
-\frac{\hbar^{2}}{2 m} \frac{\partial^{2}}{\partial x^{2}} \Psi(x, t)+U(x) \Psi(x, t)=-\frac{\hbar}{i} \frac{\partial}{\partial t} \Psi(x, t)
$$

The left side of this equation can be rewritten using the Hamiltonian operator (or total energy operator).

$$
H=-\frac{\hbar^{2}}{2 m} \frac{\partial^{2}}{\partial x^{2}}+U(x)
$$

Using the Hamiltonian operator ( $H$ ), formula (15) can be expressed as follows.

$$
H \Psi(x, t)=-\frac{\hbar}{i} \frac{\partial}{\partial t} \Psi(x, t)
$$

Since the Schrödinger equation is a partial differential equation, the product method can be used to separate the equation into spatial and temporal parts.

The wave function $\Psi(x, t)$ that describes the quantum state in time for the wave equation for the change is to make the particles are described as follows.

$$
i \hbar \frac{\partial}{\partial t} \Psi(\vec{r}, t)=\left\{-\frac{\hbar^{2}}{2 m} \nabla^{2}+V(\vec{r})\right\} \Psi(\vec{r}, t)
$$




\subsection{SchrÖdinger equation in the spherical coordinate system}

If the potential of the physical system to be examined is spherically symmetric, then the Schrődinger equation in spherical polar coordinates can be used to advantage. For a three dimensional problem, Laplacian in spherical polar coordinates is used to express the Schrődinger equation in the condensed form

$$
\frac{1}{r^{2}} \frac{\partial}{\partial r}\left(r^{2} \frac{\partial}{\partial r} \Psi\right)+\frac{1}{r^{2} \sin \theta} \frac{\partial}{\partial \theta}\left(\sin \theta \frac{\partial}{\partial \theta} \Psi\right)+\frac{1}{r^{2} \sin ^{2} \theta} \frac{\partial^{2} \Psi}{\partial \varphi^{2}}-\frac{2 m}{\hbar^{2}}(V(r)-E) \Psi=0
$$

\section{Hamiltonian based in The Classical Electromagnetic Wave}

In this section, we accomplish to substitute the quantum mechanical momentum for the classical. Here, we can think Lorentz gauge and Coulomb gauge about the electromagnetic wave.

In classical physics, gauge transformation is the in variance of the fields[15-16]. When the charged particle (electron ; e) moves in an electric field, the particle is taken to the Lorentz force.

$$
\vec{F}=e\left(\vec{E}+\frac{\vec{v}}{c} \times \vec{B}\right)
$$

In case of the absence of electromagnetic fields, the Hamiltonian that describes the movement of the charged particles of mass $m$ is

$$
H=\frac{\hat{p}^{2}}{2 m}
$$

The $\hat{p}$ and $H$ are expressed within electromagnetic field,

$$
\begin{aligned}
& \hat{p} \rightarrow \hat{p}-\frac{e}{c} \vec{A} \\
& H \rightarrow H-e \phi
\end{aligned}
$$

Therefore, Hamiltonian is

$$
H=\frac{1}{2 m}\left(\hat{p}-\frac{e}{c} \vec{A}\right)^{2}+e \phi
$$

From the Hamiltonian, Schrödinger Equation is

$$
i \hbar \frac{\partial \Psi(\vec{r}, t)}{\partial t}=\frac{1}{2 m}\left[\left(\frac{\hbar}{i} \nabla-\frac{e}{c} \vec{A}(\vec{r})\right)^{2}+e \phi(\vec{r})\right] \Psi(\vec{r}, t)
$$

If the Schrödinger equation were invariant by gauge transformation, wave equation $\Psi(\vec{r}, t)$ is phase transformation. Therefore, we were supposed to be able to phase transformation $\Psi(\vec{r}, t)$.

$$
\psi(\vec{r}, t)=e^{-\frac{i e}{\hbar c} \lambda(\vec{r}, t)} \Psi(\vec{r}, t)
$$

If charged particles were moved in the electromagnetic field, we have to accomplish gauge transformation in order to have the same physical meaning Schrődinger equation. Therefore, Schrődinger equation is

$$
i \hbar \frac{\partial \psi(\vec{r}, t)}{\partial t}=\left[\frac{1}{2 m}\left(\frac{\hbar}{i} \nabla-\frac{e}{c} \vec{A}\right)^{2}+e \phi\right] \psi(\vec{r}, t)
$$

\section{Hamiltonian based on The Poynting Vector and Analysis of Dipole Antenna by The Maxwell-Schr dinger Equation}

Antenna radiation characteristics are converted into spherical coordinates. So, it is important that antenna propagation analysis represents using the spherical system of the Schrödinger equation [11-14]. Quantized Hamiltonian of electromagnetic waves is electromagnetic waves with an average pointing vector [12-14].

$$
H=\frac{1}{2} \int_{V}\left(\varepsilon_{0}|E|^{2}+\mu_{0}|H|^{2}\right) d V
$$

Therefore, when the propagation produces total system energy, the dipole antenna with radiation can be expressed as follows. 


$$
E_{\text {total }}=\frac{I_{0}{ }^{2} \eta}{8 \pi^{2} r^{2}} \times\left[\sin \theta \frac{\cos \left(\frac{k l}{2} \cos \theta\right)-\cos \left(\frac{k l}{2}\right)}{1-\cos ^{2} \theta}\right]^{2}
$$

Finally the dipole antenna using the Schrödinger equation can be expressed as follows.

$$
\begin{aligned}
& \frac{1}{r^{2}} \frac{\partial}{\partial r}\left(r^{2} \frac{\partial}{\partial r} \Psi\right)+\frac{1}{r^{2} \sin \theta} \frac{\partial}{\partial \theta}\left(\sin \theta \frac{\partial}{\partial \theta} \Psi\right)+\frac{1}{r^{2} \sin ^{2} \theta} \frac{\partial^{2} \Psi}{\partial \varphi^{2}} \\
& \left.\left.\quad=\frac{2 m}{\hbar^{2}}\left[\frac{\mu I_{0}}{4 \pi} \int_{-1 / 2}^{+1 / 2 \sin \left[\frac{k(l / 2)-|z|}{2} \mid\right]} e^{-i / k} d z^{\prime}-\frac{I_{0}^{2} \eta}{8 \pi^{2} r^{2}} \times \sin \theta \frac{\cos \left(\frac{k l}{2} \cos \theta\right)-\cos \left(\frac{k l}{2}\right)}{1-\cos ^{2} \theta}\right]\right]^{2}\right] \Psi
\end{aligned}
$$

Where, $\Psi$ is need to revise $\Psi_{E}, \Psi_{H}$ to accomplish E-filed and $\mathrm{H}$-field.

$$
\begin{aligned}
& \Psi_{E}=\Sigma\left(i \eta \frac{I_{0} e^{-i k r}}{2 \pi r} \sin \theta \frac{\cos \left(\frac{k l}{2} \cos \theta\right)-\cos \left(\frac{k l}{2}\right)}{1-\cos \theta}\right) \\
& \Psi_{H}=\Sigma\left(i \frac{I_{0} e^{-i k r}}{2 \pi r} \sin \theta \frac{\cos \left(\frac{k l}{2} \cos \theta\right)-\cos \left(\frac{k l}{2}\right)}{1-\cos \theta}\right)
\end{aligned}
$$

and $m$ is mass of electron.

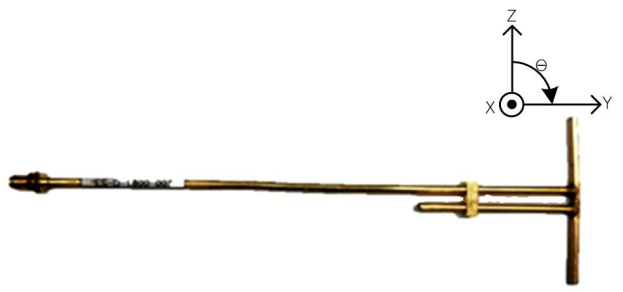

\section{$123+567 \times 91011120$ is is 16 is is 19202122232425}

[Fig. 2] Fabricated Conventional Dipole Antenna

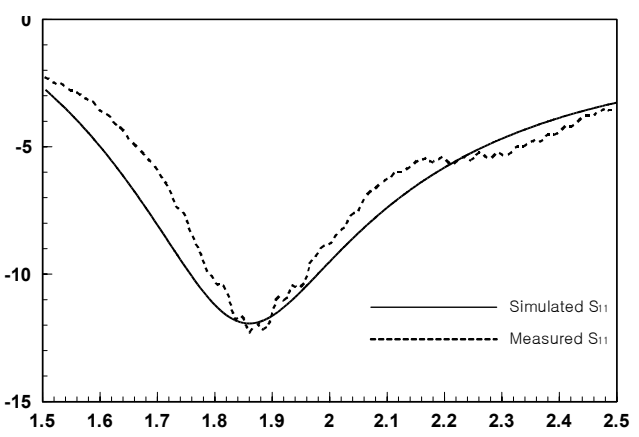

[Fig. 3] Measured and Simulated S-parameter of the Dipole Antenna.

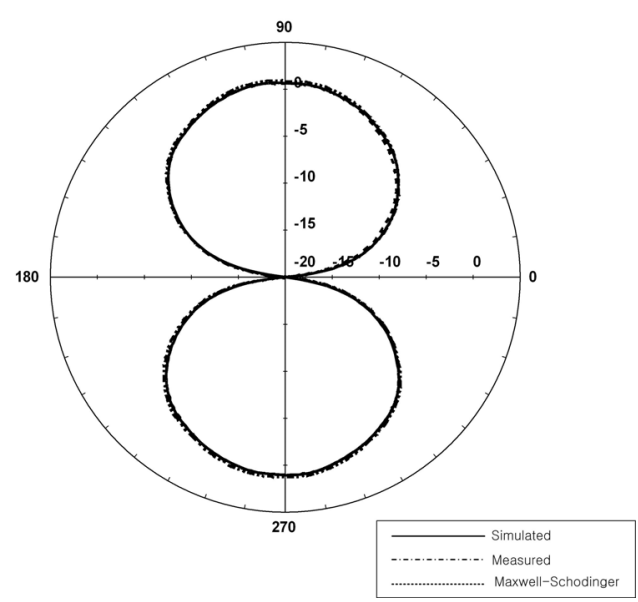

(a)

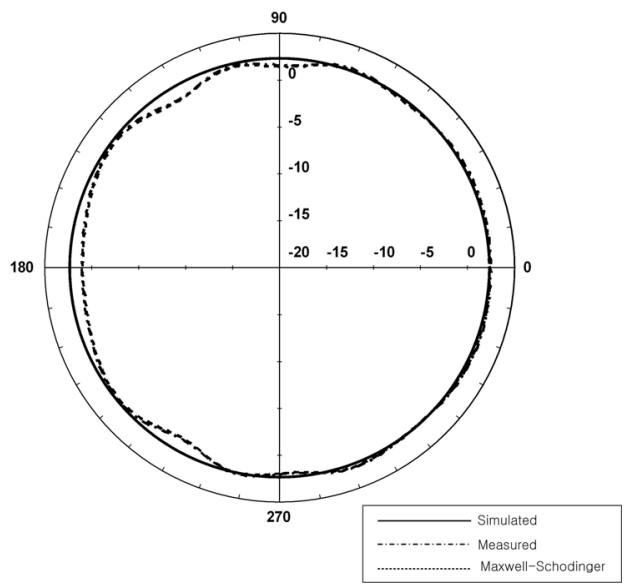

(b)

(a) E-Field Pattern (@1.85GHz_Horizenal)

(b) H-Field Pattern (@1.85GHz_Vertical)

[Fig. 4] E-H field pattern pattern of the Dipole Antenna (@1.85GHz) parameter of the Dipole Antenna.

\section{Demonstration of Maxwell-Schr dinger Equation}

The equation (32) is existing and other expression on the propagation of the dipole antenna. This is combination type of Maxwell's equation and Schrödinger equation. So as to prove equation (32), we proposed dipole antenna, as shown in Fig.1 and each of the length information is summarized in table.1. Also, 
Fig. 2 is fabricated conventional dipole antenna. The simulated and measured return losses (S11) of conventional dipole antenna are shown in Fig.3. In order to derive equation (32), we accomplish E-plane and $\mathrm{H}-$ plane plot in the center frequency (@ 1.85). If you plot the equation (32) at the resonance frequency (@1.85GHz), you will be expressed as Fig.4. As shown, the E-plane and $\mathrm{H}$-plane patterns which are very similar to simulation, measurement, and induced combining the Maxwell equation and the Schrödinger equation that is Maxwell-Schrödinger equation.

\section{Conclusion}

The Maxwell equation is fundamental in interpreting the movement of electromagnetic waves. The Schrödinger equation is based on treating wave movements that generate particles during exercise. In this paper, to analyze the dipole antenna, its propagation is derived using a formula that combines the Maxwell and Schrödinger equations. To verify the equation, the $\mathrm{E} / \mathrm{H}$-fields were plotted in the far field. As a result, the derived $\mathrm{E} / \mathrm{H}$-field patterns of the Maxwell-Schrödinger equation formula and the $\mathrm{E} / \mathrm{H}$-field patterns of measurement are similar. In the future, it will be possible to fabricate antennas using non-linear material.

\section{References}

[1] S. Li, Z. Yu, S. F. Yen, W. C. Tang, and P. J. Burke, "Carbon nanotube transistor operation at 2,6 GHz”, Nano Lett, vol. 4, no. 4, pp. 753-756, 2004.

DOI: http://dx.doi.org/10.1021/n10498740

[2] G.W. Hanson, "Fundamental transmitting properties of carbon nanotube antennas," in IEEE Int. Symp. Antennas Propagation, Washington, DC, Jul. 2-3, 2005.

DOI: http://dx.doi.org/10.1109/APS.2005.1552484

[3] G.Fikioris, "On the application of numerical methods to Hallén's equation," IEEE Trans Antennas Propag., vol. 49, pp.383-392, Mar.2001

DOI: http://dx.doi.org/10.1109/8.918612

[4] C. A. Balanis, "Pattern Distortion due to Edge Diffractions," IEEE Trans Antennas propag., vol. 18, pp.561-563, July. 1970

DOI: http://dx.doi.org/10.1109/TAP.1970.1139737

[5] J. Huang, "The finite ground plane effect on the microstrip antenna radiation patterns", IEEE Trans Antennas Propag., vol. 31, no. 4, July 1983. DOI: http://dx.doi.org/10.1109/TAP.1983.1143108

[6] K. S. Yee, "Numerical solution of initial boundary value problems involvingMaxwell's equations in isotropic media," IEEE Trans. Antennas Propagat., vol. AP-14, pp. $302-307$, May 1966.

DOI: http://dx.doi.org/10.1109/TAP.1966.1138693

[7] C. A. Balanis, Antenna Theory: Analysis and Design,2nd ed. New York: Wiley, 1997

[8] Namki. T, "A new FDTD algorithm based on alternating-direction implicit method," IEEE Trans. Microwave Theory and Technniues., vol. 47, no.10, pp. 2003-2007, Oct 1999.

DOI: http://dx.doi.org/10.1109/22.795075

[9] H.R Hassani, M.Jahanbakht, "Method of moment analysis of finite phased array of aperture coupled circular microstrip patch antennas", Progress In Electromagnetics Research B, vol. 4, pp. 197-210, 208.

[10] Klaus-Jurgen Bathe, Finite Element Method,

[11] S.Salahuddin, M.Lundstrom, and S. Datta, "Transport effects on signal propagation in quanum wires," IEEE Trans. Electron devices, vol. 52, no. 8, pp. 1734-1742, Aug. 2005

DOI: http://dx.doi.org/10.1109/TED.2005.852170

[12] Amit Gowami, Quantum mechanics, WBC, 1972

[13] Richard L. Liboff, Introductory quantum mechanics, 4nd ed, Addison Wesley, 2003

[14] Y. Aharonov, D. Bohm, "Significance of Electromagnetic Potentials in the Quantum Theory," Phys. Rev, vol. 115 no.3, pp. 485-491, Aug. 1959. DOI: http://dx.doi.org/10.1103/PhysRev.115.485

[15] Ya. B. Zel'dovich, "Scattering and emission of a quantum system in a strong electromagnetic wave," Sov. Phys.-Usp., Vol. 16, No. 3, November-December 1973.

DOI: http://dx.doi.org/10.1070/PU1973v016n03ABEH005192

[15] R. W Ziolkowski, Ioannis M. Besieris, Amr M. Shaarawi, "Aperture realizations of exact solutions to homogeneous-wave equations." J. Math. Phys. Vol. 
10, No.1, Jan 1993.

DOI: http://dx.doi.org/10.1109/APS.1992.222001

\section{Jin Young Kim}

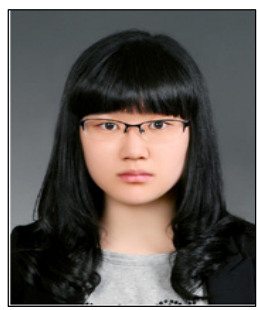

- Feb. 2010 : Chungju University Department of Nano Science (Undergraduate)

- Sep. $2011 \sim$ current : Seoul National University of Science and Technology, Graduate School of NID Fusion Technology, M.S. student

$<$ Research Interests $>$

Frequency Selective Surface(FSS), EM theory.

\section{Jae-Young Chung}

[Regular member]

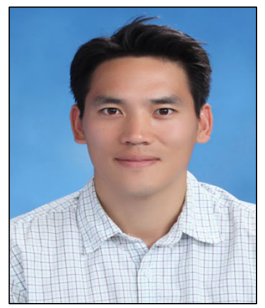

- Feb. 2002 : Yonsei University, Department of Electronics Engineering (Undergraduate)

- Mar. 2007 : Ohio University, Electrical Engineering and Computer Science (M.S)

- Jun. 2010 : Ohio University, Electrical Engineering and Computer Science (Ph.D)

- Jun. 2002 Jun. 2004 : Motorola Korea, Research Engineer

- Jun. 2010 Aug. 2012 : Samsung Electronics, Principal Engineer

- Sep. 2012 current : Seoul National University of Science and Technology, Department of Electrical and Information Engineering, Assistant Professor

$<$ Research Interests $>$

Antenna design and measurement
Young-Bae Jung

[Regular member]

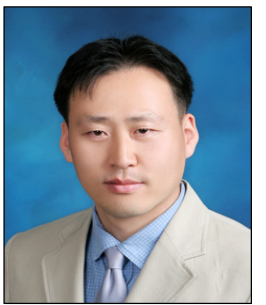

- Feb. 2001 : Information and Communication University (ICU), Electrical Engineering (M.S)

- Aug. 2009 : KAIST, IT Engineering (Ph.D)

- Feb. $2001 \sim$ Feb. 2012 : ETRI, Radio Science Department, Principal Engineer

- Mar. $2012 \sim$ current : Hanbat University, Department of Electrical and Control Engineering, Assistant Professor

$<$ Research Interests $>$

Antenna, RF/Microwave, Mobile/Satellite communication

\section{Chang Won Jung}

[Regular member]

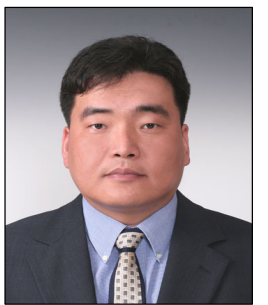

- Dec. 2001 : University of Southern California, Electrical Engineering (M.S)

- Jun. 2005 : University of California, Irvine, Electrical Engineering (Ph.D)

- Jan. 1997 Jun. 2000 : LG, Electronics, Research Engineer

- Jul. 2005 Oct. 2005 : University of California, Irvine, Post Doctor

- Nov. $2005 \sim$ Apr. 2008 : Samsung Advanced Institute of Technology, Principal Research Engineer

- May $2008 \sim$ current : Seoul National University of Sceince and Technology, Graduate School of NID Fusion Technology, Assistant Professor

$<$ Research Interests $>$

Antenna, RF, EMI/EMC, RF-MEMS, Sensor 